abnormal organic aciduria dicarboxylic acidurea hypoglycemia hypoglycine metabolite analogue Jamaican vomiting sickness

Reye's-like syndrome

\title{
Twin Siblings with a Reye's-Like Syndrome Associated with an Abnormal Organic Aciduria, Hypoglycemia, Diarrhea, and Vomiting with Close Similarities to Jamaican Vomiting Sickness
}

\author{
RONALD A. CHALMERS. ${ }^{1391}$ ALEXANDER M. LAWSON, ANDREW WHITELAW, AND PAUL \\ PURKISS \\ Divisions of Inherited Metabolic Diseases [R.A.C.. P.P./ and Clinical Chemistry [A.M.L.] and Department of \\ Padiatrics [A.W.J. Northwick Park Hospital, and M. R. C. Clinical Research Cenire. Watford Road, Harrow, HAI \\ 3UJ, United Kingdom
}

\begin{abstract}
Summary
Seventeen-month-old identical Caucasian male twins developed vomiting and diarrhea, and both were admitted to hospital $30 \mathrm{hr}$ after the onset of symptoms. One was dead on arrival. Hepatic histology showed fatty degeneration affecting all parts of the lobule. Death was due to aspiration of gastric contents. Electron microscopic examination of the intestine showed the presence of replicating adenovirus. The other infant was comatose with undetectable blood glucose. He was maintained on IV glucose for $48 \mathrm{hr}$ and made a complete recovery with no further hypoglycemia even after an overnight fast. Eighteen months later, he was a normally developed chilc for his age. The clinical diagnosis made in both infants was Reye's syndrome.

Urinary organic acids were determined by gas chromatography and identified by gas chromatography-mass spectrometry. Greatly increased concentrations of adipic acid, 4-hydroxyphenylacetic acid, suberic acid, and of a previously unrecorded urinary organic acid which has been identified as 5-hydroxyhexanoic acid were observed. There were also increased concentrations of octanoic, sebacic, decenedioic, octenedioic, and 3-hydroxyisovaleric acids, and greatly reduced concentrations of the aldonic and deoxyaldonic acids and acids of the tricarboxylic acid cycle. Lactic acid excretion was not increased, and there was only a moderate increase in the concentrations of 3-hydroxybutyric and acetoacetic acids. No gross increase in concentrations or abnormal acids were observed in the urinary volatile short chain fatty acids.

The organic acids observed in the urine of the present patients have been compared to those observed in patients with Jamaican vomiting sickness and those with Reye's syndrome. The similar dicarboxylic $C_{6}-C_{10}$ aciduria and profound hypoglycemia observed in the present patients and in those with Jamaican vomiting sickness is of interest and probably occurs due to a similar mechanism, inhibition of $\boldsymbol{\beta}$-oxidation of medium-chain fatty acids by a chemical toxin. Some differences between the organic aciduria observed in these cases and in patients with Jamaican vomiting sickness suggest that the nature of the possible toxin, although closely similar, is not identical. The occurrence in both of the present patients of high concentrations of 5-hydroxyhexanoic acid may indicate the nature of the causative xenobiotic agent, and it has been suggested that this could be hex-4-enoic acid.
\end{abstract}

\section{Speculation}

The occurrence of profound hypoglycemia and abnormal organic aciduria in twin English siblings with a Reye's-like syndrome and close clinical and biochemical similarities to Jamaican vomiting sickness suggests that some cases of Reye's syndrome in infants are due to causes similar to that involved in Jamaican vomiting sickness, that is, organic acid chemical toxins related to metabolites of hypoglycin.

Encephalopathy and fatty degeneration of the viscera in infants and children (Reye's syndrome) has been widely reported since the original description by Reye et al. (27), and a consistent clinical, biochemical, and pathological picture has evolved from these reports $(12,22)$. There is a considerable variation, however, in the incidence, presentation, and severity of the disease, and the etiology, particularly in infants, is unclear $(17,22)$. It has been suggested that the syndrome may represent the effects of many different etiologic factors acting on the same or common metabolic pathways and thereby leading to similar results. The similarities of Reye's syndrome and the vomiting sickness of Jamaica (ackee poisoning or hypoglycin toxicity) have also been noted (21). Tanaka et al. (30) have reported the biochemical investigation of two siblings with Jamaican vomiting sickness and included detailed organic acid analyses that confirmed the cause of the disease and also the dissimilar findings in patients with Reye's syndrome. The recent presentation of identical twin siblings with an unusual Reye's-like syndrome $(8,36)$, enabled a detailed study to be made of the urinary organic acids in the disease that show close similarities to those reported in Jamaican vomiting sickness that provide information on the biochemical mechanisms of the disease and of the possible underlying etiology in these cases. This paper presents the detailed case reports and biochemical studies on the abnormal organic aciduria in these infants.

\section{CASE REPORT}

Seventeen-month-old identical Caucasian male twins, R. B. and T. B., developed vomiting and diarrhea on October 30, 1976. Their mother treated them both with plain water, one $75 \mathrm{mg}$ aspirin tablet every $12 \mathrm{hr}$, and $5 \mathrm{ml}$ of Kaolin mixture. They became drowsy, and $30 \mathrm{hr}$ after the onset of symptoms, R. B. was found blue and cold in his bed. Both twins were brought to Northwick Park Hospital immediately by ambulance.

\section{PAST MEDICAL HISTORY AND FAMILY HISTORY}

There was an uneventful twin pregnancy with vaginal delivery at $38 \mathrm{wk}$. Birth weight for both was $2.2 \mathrm{~kg}$. The twins spent two 
wk in the Special Care Baby Unit because they were initially slow to feed. Urinary organic acids examined at this time were entirely normal. Subsequent growth and development were normal. The parents are nonconsanguinous and healthy. The sister, aged $4 \frac{1}{2}$ years, had diarrhea and vomiting 2 wk previously.

\section{CLINICAL COURSE}

R. B. was dead on arrival at the hospital. Blood culture was sterile, but a throat swab grew $\beta$-hemolytic streptococci. Autopsy revealed some hepatic enlargement. Histology showed fatty degeneration affecting all parts of the lobule. The lungs showed intense vasocongestion, but no pneumonia, the appearances being similar to those seen in aspiration of gastric contents. Brain histology was normal. Electron microscopic examination of the intestine (Heather A. Davies) showed adenovirus particles within the nucleus of a mucosal cell and in scrapings of the mucosa. The intranuclear virus particles were in crystalline array, indicating virus replication. There was no virus in the liver, lung. or brain. There was no intestinal lymphoid nodular hyperplasia.

T. B. was comatose on arrival, responding sluggishly to painful stimuli and with brisk tendon reflexes. He began to convulse and was given IM paraldehyde immediately. A Detrostix showed an unrecordable blood glucose [later confirmed at $0.2 \mathrm{mmoles} / \mathrm{liter}$ $(4 \mathrm{mg} / 100 \mathrm{ml})]$. After IV injection of $5 \mathrm{~g}$ of glucose, his clonic movements stopped, and he sat up. Investigation at the time of admission showed: blood glucose, $0.2 \mathrm{mmole} / \mathrm{liter}(4 \mathrm{mg} / 100 \mathrm{ml})$; plasma urea, $13.5 \mathrm{mmoles} / \mathrm{liter}(88 \mathrm{mg} / 100 \mathrm{ml})$; bicarbonate, 11 mmoles/liter; serum insulin, 48 munits/liter (raised); plasma cortisol, 1460 nanomoles/liter (appropriate); growth hormone, 69 munits/liter (appropriate); aspartate transaminase, $208 \mathrm{IU} /$ liter (raised). Plasma calcium, sodium, potassium, bilirubin, and alkaline phosphatase were normal. He was maintained on IV glucose for the next $24 \mathrm{hr}$, blood glucose levels being around 6 mmoles/ liter $(120 \mathrm{mg} / 100 \mathrm{ml})$. When IV glucose was discontinued, he became drowsy and hyperreflexic again and the blood glucose fell to $1.9 \mathrm{mmoles} / \mathrm{liter}(36 \mathrm{mg} / 100 \mathrm{ml})$. Glucagon, $1 \mathrm{mg} \mathrm{IM}$, raised the blood glucose to $4.2 \mathrm{mmoles} / \mathrm{liter}(76 \mathrm{mg} / 100 \mathrm{ml})$, and IV glucose was given for a further $24 \mathrm{hr}$. He made a complete recovery with no further hypoglycemia even after an overnight fast and had a normal fasting serum insulin level. Eighteen months later, he was a normally developed child for his age with a tendency to upper respiratory infections and a low serum IgA.

\section{MATERIALS}

Postmortem urine was obtained from R. B. on admission by suprapubic aspiration, and urine collections were made from $T$. B. on admission and on days $I$ to 2 , and 4 , and follow-up on day 37 after admission. Normal control data were obtained on urine specimens collected from 20 infants in the same age groups, and comparison was also made to specimens from over 650 neonates and infants with acute illness of other causes. Other specimens were taken, and investigations were made as described in the case reports.

\section{METHODS}

Urinary organic acids were determined by gas chromatography using previously described methods $(9,16,19)$ that involve quantitative extraction by anion-exchange chromatography onto DEAE-Sephadex, stabilization of oxo acids as $O$-ethyloximes, and freeze-drying under controlled conditions of $-10^{\circ} \mathrm{C}$ and 0.5 torr. The dry residue was then either trimethylsilylated with minimum quantity of $\mathrm{N}, \mathrm{O}$-bis(trimethylsilyl)trifluoroacetamide or reconstituted in $2 \mathrm{ml}$ water for further extraction with solvents. In the latter procedure, which is designed to examine in more detail the more hydrophobic acidic metabolites, the reconstituted extract is saturated wih solid sodium chloride, acidified to below $\mathrm{pH} 2$ with hydrochloric acid, and extracted successively three times with diethyl ether and three times with ethyl acetate. The solvent extracts are combined without drying to avoid loss of acids in the water component. blown down to dryness at room temperature with a stream of dry nitrogen, and trimethylsilylated as above.

The individual organic acids were identified using gas chromatography-mass spectrometry including repetitive scanning and maximizing ion techniques as described elsewhere (7). Before gas chromatography-mass spectrometry, trimethylsilyl-sulphate was removed from the DEAE-Sephadex extracts by chromatography on Lipidex 5000 (34). Completely unknown acids were identified with the aid of high-resolution mass spectrometry and by comparison to synthesized standards as detailed elsewhere (6).

Amino acids were determined by quantitative ion-exchange chromatography with a Technicon TSM analyzer.

\section{RESULTS}

Urinary and blood amino acids were normal except for increased levels of urinary $\beta$-aminoisobutyric acid which did not persist as the patient recovered. This amino acid is associated with tissue catabolism.

Figure 1 illustrates the chromatogram of urinary organic acids in the specimen of urine from T. B. collected during the day after admission. The upper part of Figure 2 shows the computer plot of the summed ion intensity or total ionisation plot versus scan number for the sample (desulphated) illustrated in Figure 1. This plot closely resembles the gas chromatogram (Fig. 1) obtained with a conventional flame ionization detector. The lower part of Figure 2 is of the summed maximizing ion intensity plot versus scan number and illustrates the large increase in apparent resolution which can be achieved by applying the "maximizing ion" criteria (7), for example in the areas of peak numbers 18, 19, 20. and 21 . Peak numbers in Figures 1 and 2 correspond, and identifications are given in the legend to Figure 1. The chromatograms illustrate the greatly increased concentration of adipic acid (peak 10), 4-hydroxyphenylacetic acid (peak 14), suberic acid (peak 16), and of a previously unrecorded urinary organic acid (peak 8) which has been identified as 5 -hydroxyhexanoic acid (6). There were also increased concentrations of octanoic, sebacic, decenedioic, and octenedioic acids and greatly reduced concentrations of the aldonic and deoxyaldonic acids (19) and of acids of the tricarboxylic acid cycle. Lactic acid excretion was not increased. and there was only a moderate increase in the concentration of 3 hydroxybutyric and acetoacetic acids.

Figure 3 illustrates the conventional gas chromatogram of the more hydrophobic acids in the same urine specimen obtained by solvent reextraction of the reconstituted DEAE-Sephadex extract. Peak identifications are given in the legend to Figure 3. This extract enables a more detailed study of the minor acidic components in the urine, allowing, for example, the identification and semiquantitation of additional previously unrecorded hydroxyhexanoic acids. 3-methylglutaconic acid, 2-hydroxyglutaric acid, pimelic acid, and salicyluric acid, which are otherwise obscured by other components or present at very low levels in the urine.

Table 1 records the quantitative urinary concentrations of the major organic acids observed in both $R$. B. and $T$. B. at the time of admission and in T. B. on subsequent days and compared to the normal levels of excretion observed in infants, together with 24-hr excretions of major acidic metabolites in T. B.

No gross increase in concentration or abnormal acids were observed in the urinary volatile short-chain fatty acids in T. B. No acylglycines were observed in any of the extracts.

\section{DISCUSSION}

Reye's syndrome has been associated with a number of viruses, for example, influenza $B$ virus in older children (11) and in infants is almost always preceded by a mild illness, often accompanied by diarrhea (17). Vomiting is a less constant feature, and coma, seizures, and respiratory disturbances are the most frequent clinical manifestations. The incidence in infants is unknown, and 


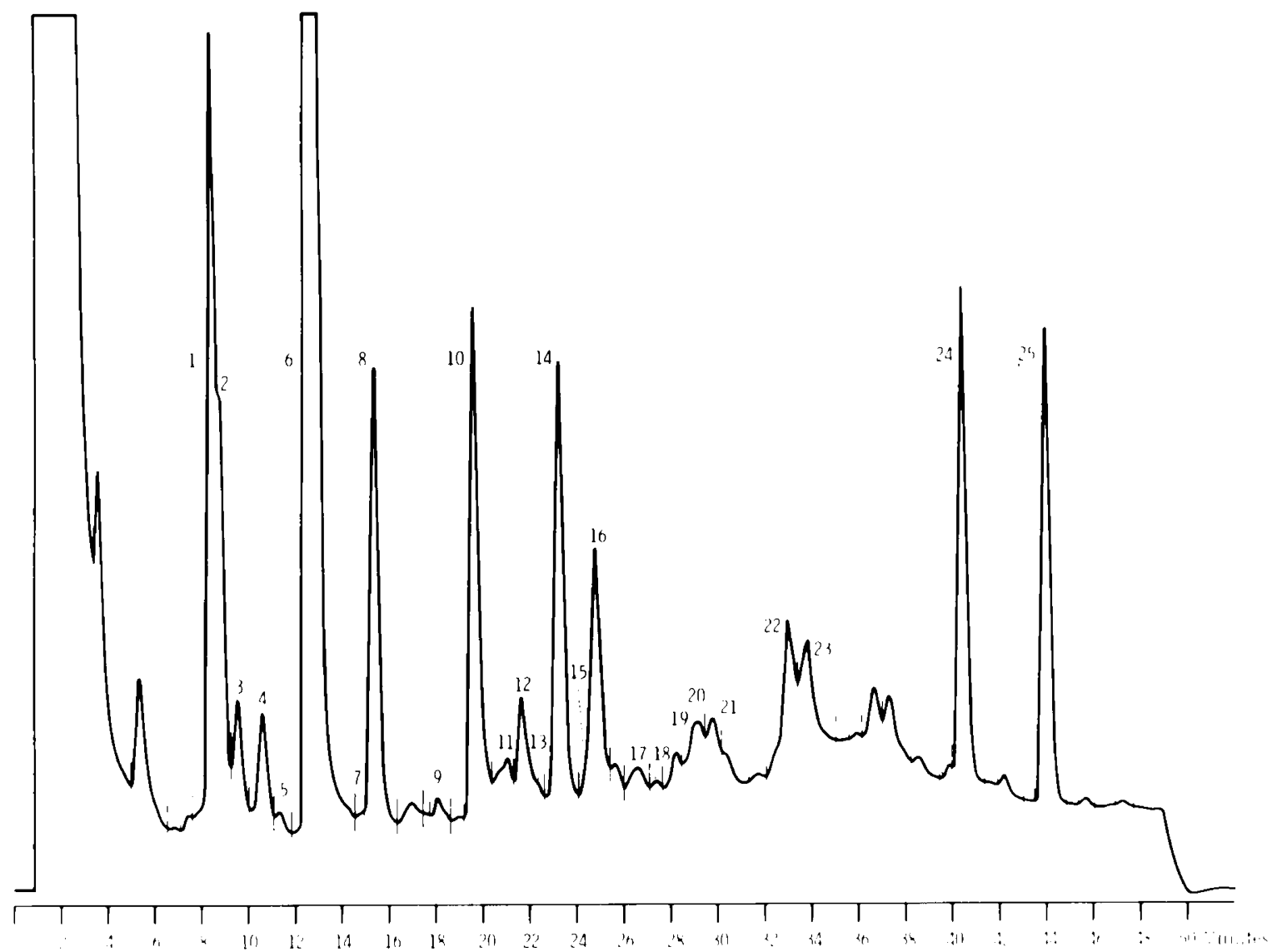

Fig. 1. (jas chromatogram of urinary organic acids from T. B. (day after admission) obtained by DEAE-Sephadex extraction. Separated as their trimethylsilyl and trimethylsilyl ethoximes by gas chromatography on $1.83 \times 0.003 \mathrm{~m}$ glass columns packed with $10 \%$; OV 101 on High Performance (hromosorb W, 80 to 100 mesh, by temperature programming from 110 to $285^{\circ} \mathrm{C}$ at $4^{\circ} \mathrm{C} / \mathrm{min}$ with a 5 -min initial isothermal delay. Peak identifications are: 1, sulphate: 2, 3-hydroxybutyric acid: 3. acetoacetic acid: 4. 3-hydroxyisovaleric acid: 5. cotanoic acid: 6 , phosphate: 7, glyceric acid: 8,5 hydroxyhexanoic acid; 9, 2-deoxytetronic acid: 10, adipic acid: 11 , methyladipic acid: 12, tetronic acids: 13, 3-hydroxy-3-methylglutaric acid: 14, 4hydroxyphenylacetic acid: 15 , octenedioic acid: 16 , suberic acid: 17 . homovanillic acid $(+), 18$, glycerophosphoric acid: 19 . pentonic acids: 21 , decenedioic acid: 21 , sebacic acid: 22, glucuronic acid: 23, glucaric acid: 24, $n$-tetracosane (internal standard): 25, n-hexacosane (internal standard).

many cases probably remain undiagnosed (17). The outcome of Reye's syndrome in infants is generally poor, and residual neurologic deficits often occur (35). The syndrome is uncommon in siblings, although familial occurrences, including at least one in twin infants (33), have been reported (17). The absence of familial occurrences tends to negate the suggested genetic susceptibility towards Reye's syndrome in some infants with prodomal infections (2). Encephalopathy and fatty degeneration of the viscera also characterize specific diseases in which chemical toxins have been identified or implicated including Jamaican vomiting sickness, mycetismus, and aflatoxin ingestion (10, 24). It has been suggested that these agents act as mitochondrial toxins, and it has been demonstrated that viruses may potentiate the action of similar synthetic toxins (10), for example pent-4-enoic acid, an analogue of the active metabolite of hypoglycin. methylenecyclopropylacetic acid. A xenobiotic origin of Reye's syndrome has been suggested in line with these findings (23). with the toxin causing mitochondrial metabolic disruption of differing severity dependent on the toxin concerned, which is perhaps potentiated in many cases by viral infections $(10)$. It is of note. however. that Mullen (23) has suggested that the exposure to the toxin occurs prior to the viral agent. causing subclinical damage, altered lipid metabolism, and impaired immune responsiveness, leading to susceptibility to viral infection. It is of interest in this context that the liver appearances in $\mathrm{R}$. B. indicated a lesion of some time.

Similarities between Reye's syndrome and Jamaican vomiting sickness are frequently indicated (10, 21, 22), but discrete and fundamental differences also occur. Table 2 compares the reported features of these two syndromes with the observations in the present cases that show closer similarities to Jamaican vomiting sickness than to Reye's syndrome. As indicated by Table 2, the present cases were associated with replicating adenovirus in the gut (36). Adenovirus has been implicated as a cause of gastroenteritis, and adenoviruses have been isolated from children with a wintertime diarrheal illness associated with respiratory symptoms (3). Reye's syndrome has been compared with viral ketoacidosis (1). but adenovirus occurs in the gut of normal children. and the organic aciduria observed in the present cases is inconsistent with a diagnosis of gastroenteritis. ('hildren with gastroenteritis show a gross ketonuria with greatly elevated urinary concentrations of 3-hydroxybutyrate and acetoacetate without an increase in the concentration of 4-hydroxyphenylacetic acid (5). The present cases showed little evidence of ketosis and had high concentrations of 4-hydroxyphenylacetic acid in their urine, this being consistent with intestinal stasis, malabsorption. or intestinal mucosal damage, as could be expected with an ingested toxin. The other organic acids observed in the urine of the present cases provide vital information on the underlying biochemistry and suggest that the etiology of these cases is a chemical toxin producing a syndrome with close similarities to Jamaican vomiting sickness.

Table 3 compares the organic acids observed in the urine of the present infants to those observed in patients with Jamaican vomiting sickness (30) and those with Reve's syndrome $(15,30)$. The similar dicarboxylic $\mathrm{C}_{,-C_{1}} \mathrm{C}_{1 .}$ aciduria in both disorders is of interest 


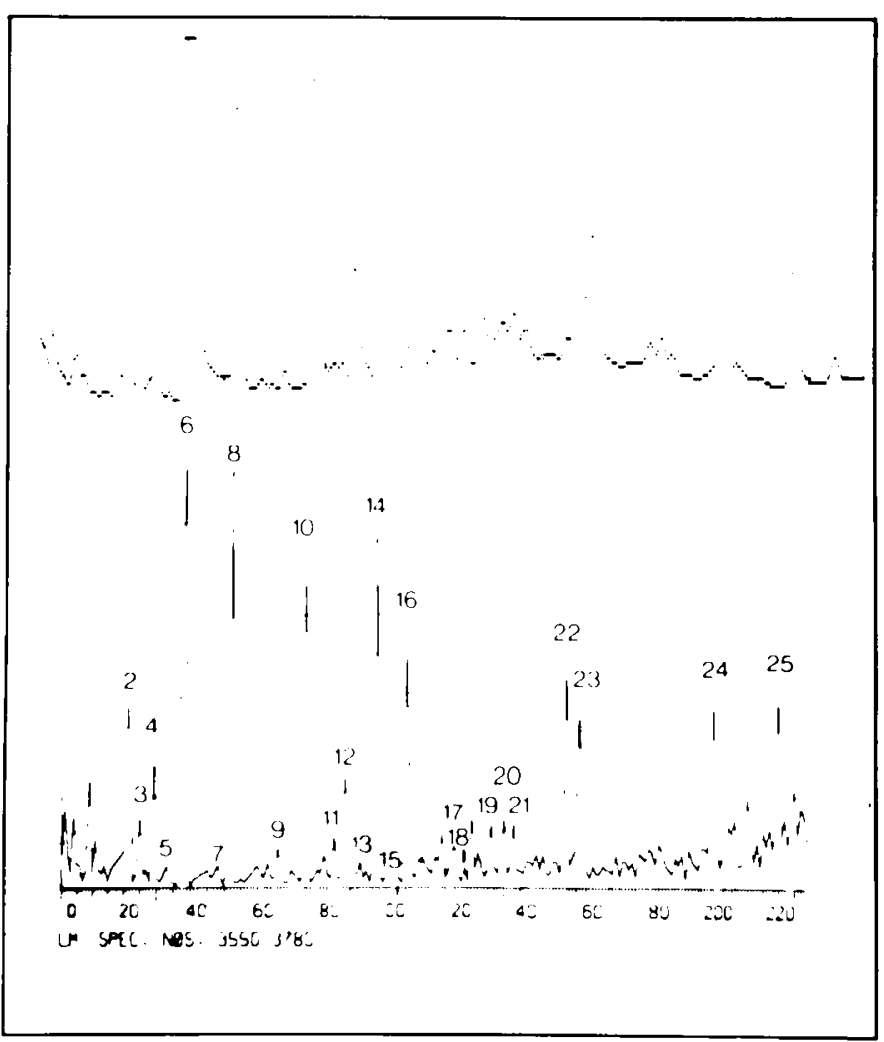

Fig. 2. Organic acids in the same urine specimen extracted illustrated in Figure 1 after desulphation on Lipidex 5000. The upper part of the figure shows the computer plot of the summed ion intensity or total ionization plot versus scan number and closely resembles the gas chromatogram obtained with conventional flame ionization detection shown in Figure 1. The lower part of the figure is of the summed maximizing ion intensity plot versus scan number and illustrates the large increase in apparent resolution obtained by this technique.

and probably occurs due to a similar mechanism. Urine from healthy infants and adults may contain low amounts of aliphatic dicarboxylic acids of chain length $C_{6}-C_{*}(4,19)$ and very low amounts of dicarboxylic acids of similar chain length containing methyl branches, cyclopropane rings, and acetylenic bonds 19 , 20 ). Increased excretion of $C_{4}-C_{10}$ dicarboxylic acids have been reported in a number of pathologic conditions including ketoacidosis, where increased concentrations of adipic and suberic acids occur (25). High concentrations of saturated and monounsaturated dicarboxylic $C_{t i}-C_{N}$ acids have been observed in patients with carnitine deficiency $(18)$ and those with possible inherited defects in the $\beta$-oxidative pathway of fatty acid metabolism (14, 20, 26, $31)$. In the present cases and in those with Jamaican vomiting sickness, there was little ketosis, and the dicarboxylic aciduria was different from that in ketotic patients. It is apparent that the mechanisms by which they are produced are probably different, with $\omega$-oxidation of long-chain fatty acids followed by normal $\beta$ oxidation in ketosis compared to inhibited $\beta$-oxidation of mediumchain fatty acids in the present cases. Jamaican vomiting sickness, and similar disorders, followed by $\omega$-oxidation to the dicarboxylic acids. Inhibition of $\beta$-oxidation occurs via inhibition of specific acyl CoA dehydrogenases and of the formation and transport of acyl carnitine esters through the mitochondrial membrane. Defective $\beta$-oxidation by inhibition of these acyl CoA dehydrogenases is also indicated in these cases by excretion of octanoic acid because this acid and the shorter chain fatty acids do not require carnitine for mitochondrial transport. There is no general increase in dicarboxylic acid excretion in Reye's syndrome $(15,30)$ indicating relatively normal $\beta$-oxidation. Further evidence of the specificity of acyl CoA dehydrogenase inhibition is given by the increased urinary concentrations of 3-hydroxyisovaleric acid, due

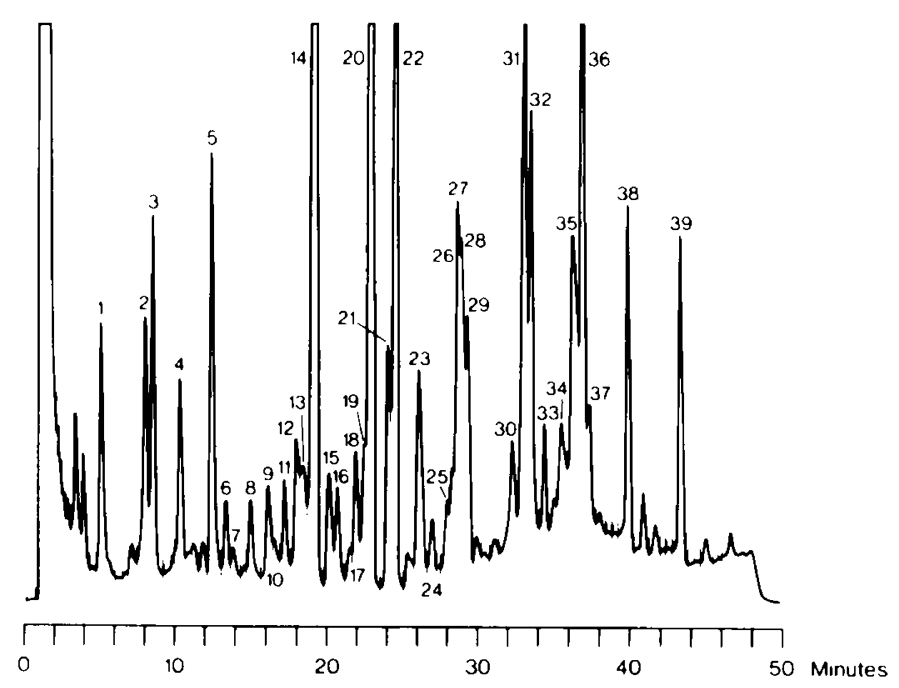

Fig. 3. Gas chromatogram of the urinary organic acids in T. B. (day after admission), obtained by solvent reextraction with ether and ethylacetate of the acidified and sodium chloride saturated reconstituted residue from the freeze-dried DEAE-Sephadex extract. Separation conditions are as described for Figure 1. Peak identifications are: 1, lactic acid + phenol: 2. cresol + 3-hydroxypropionic acid; 3. 3-hydroxybutyric acid: 4, 3-hydroxyisovaleric acid: 5 , phosphate: 6 , succinic acid: 7, 3-hydroxyhexanoic acid?: 8, 5-hydroxyhexanoic acid; 9, glutaric acid: 10. 2-methylglutaric acid?: 11, unidentified: 12, 3-methylglutaconic acid: 13, unidentified: 14, adipic acid: 15, 3-methyladipic acid: 16, trihydroxybenzene (artefact): 17 , 2-hydroxyglutaric acid: 18 , pimelic acid: 19,3-hydroxy-3-methylglutaric acid: 20,4-hydroxyphenylacetic acid: 21 , octenedioic acid; 22 , suberic acid 23, aconitic acid?: 24, unidentified, 25, hippuric acid; 26. decenedioic acid; 27 and 28 , unidentified: 29, sebacic acid + some 4-hydroxy-3-methoxymandelic acid; 31 , salicyluric acid: 34 . 4-hydroxyhippuric acid: $38, n$ tetracosane (internal standard); $39, n$-hexacosane (internal standard).

to inhibition of isovaleryl CoA dehydrogenase $(28,29)$, similar to the observations in Jamaican vomiting sickness. In contrast, however, is the absence of ethylmalonic acid, said to be characteristic of butyryl CoA dehydrogenase inhibition $(31,32)$, and only a minimal amount of glutaric acid that occurs due to inhibition of glutaryl CoA dehydrogenase (30). The lack of inhibition of butyryl CoA dehydrogenase would explain the reduced accumulation of short $C_{3}-C_{6}$ fatty acids in these patients, and although the accumulation of these acids is similar in all disorders, there were no detectable urinary mono- or dicarboxylic acid acyl glycines in the present cases. This is in contrast to the observation of hexanoylglycine in the urine of patients with Jamaican vomiting sickness (30) and a patient with proposed butyryl CoA dehydrogenase deficiency (31), indicating little or no accumulation of hexanoic acid or hexanoyl COA in the present children.

The difference in specificity of inhibition of acyl CoA dehydrogenases in these children compared to patients with Jamaican vomiting sickness indicates that the nature of the possible toxin, although closely similar, is not identical to methylenecyclopropylacetic acid, the active metabolite of hypoglycin. The occurrence in the urine of both of the present cases of high concentrations of a previously unrecorded urinary organic acid that has been identified as 5-hydroxyhexanoic acid (6) may indicate the nature of the causative xenobiotic agent. 5-Hydroxyhexanoic acid would conceivably occur due to $\omega-1$ oxidation of accumulating hexanoic acid (6), but this seems unlikely, unless these infants represent a peculiar genetic entity compared to the several reported cases of dicarboxylic aciduria with accumulation of hexanoic acid (14, 18, $25,30,31)$, none of whom apparently excreted detectable 5hydroxyhexanoic acid. The possible alternative origins of this acid are therefore of particular interest, and it has been suggested (6) that this acid could be derived from its immediate metabolic precursor hex-4-enoic acid, an analogue of methylenecyclopropylacetic acid and pent-4-enoic acid. 
Table 1. Concentrations of urinary organic acids (extracted by DEAE-Sephadex) (mg/g creatinine)

\begin{tabular}{|c|c|c|c|c|c|c|}
\hline Acid & $\begin{array}{l}\text { R. B. (postmor- } \\
\text { tem urine) }\end{array}$ & $\begin{array}{c}\text { Day I-2 } \\
\text { (IV glucose) }\end{array}$ & $\begin{array}{c}\text { Day } 4 \\
\text { (normal food) }\end{array}$ & $\begin{array}{c}\text { Day } 37 \\
\text { (fully recovered) }\end{array}$ & Normal ranges & $\begin{array}{c}\text { T. B. (mg/24 hr; day } \\
\text { after admission) }\end{array}$ \\
\hline $\operatorname{Lactic}(+)$ & 180 & 90 & -2 & -- & $70^{1}$ & \\
\hline 3-Hydroxybutyric & 30 & 250 & - & - & - & 39 \\
\hline Acetoacetic & - & 50 & - &.- & - & 8 \\
\hline 3-Hydroxyisovaleric & 40 & 60 & 2 & 20 & 10 & 9 \\
\hline Octanoic & - & 3 & - & - & - & 0.5 \\
\hline Glyceric & - & 3 & - & $\cdots$ & - & - \\
\hline 4-Deoxytetronic & - & - & 2 & 80 & $5-70$ & \\
\hline 5-Hydroxyhexanoic & 340 & 330 & - & - & - & 51 \\
\hline 2-Deoxytetronic & - & 10 & 10 & 80 & $2-60$ & - \\
\hline Adipic & 1340 & 410 & 50 & - & $10^{1}$ & 64 \\
\hline Methyladipic & - & 10 & & - & $45^{\prime}$ & - \\
\hline Tetronics & - & 70 & 95 & 210 & $2-210$ & $\ldots$ \\
\hline 2-Oxoglutaric & - & - & 30 & 20 & 50 & - \\
\hline 4-Hydroxyphenylacetic & 500 & 320 & 10 & 20 & 90 & 50 \\
\hline Octenedioic & $\cdots$ & 10 & - & - & - & $\ldots$ \\
\hline Suberic & 1150 & 220 & $150^{\prime}$ & & - . & 34 \\
\hline 2-Deoxypentonic & - & - & 150 & 140 & $5-75$ & - \\
\hline Aconitic & - & - & 70 & 70 & $2-80$ & $\cdots$ \\
\hline Pentonics + decenedioic & - & 50 & - & - & -- & 8 \\
\hline Pentonics + Citric & - & 10 & 70 & 1100 & $2-1000$ & - \\
\hline Sebacic $(+)$ & 350 & 10 & - & & $-\cdot$ & 1.5 \\
\hline Glucuronic & 200 & 180 & 20 & 10 & 80 & -- \\
\hline Gluconic & 80 & 50 & 40 & 10 & 60 & - \\
\hline
\end{tabular}

'Multiple peak.

, not observed

Table 2. Relationship of Reye's syndrome, Jamaican vomiting sickness (hypoglycin toxicity), and the Harrow', England cases

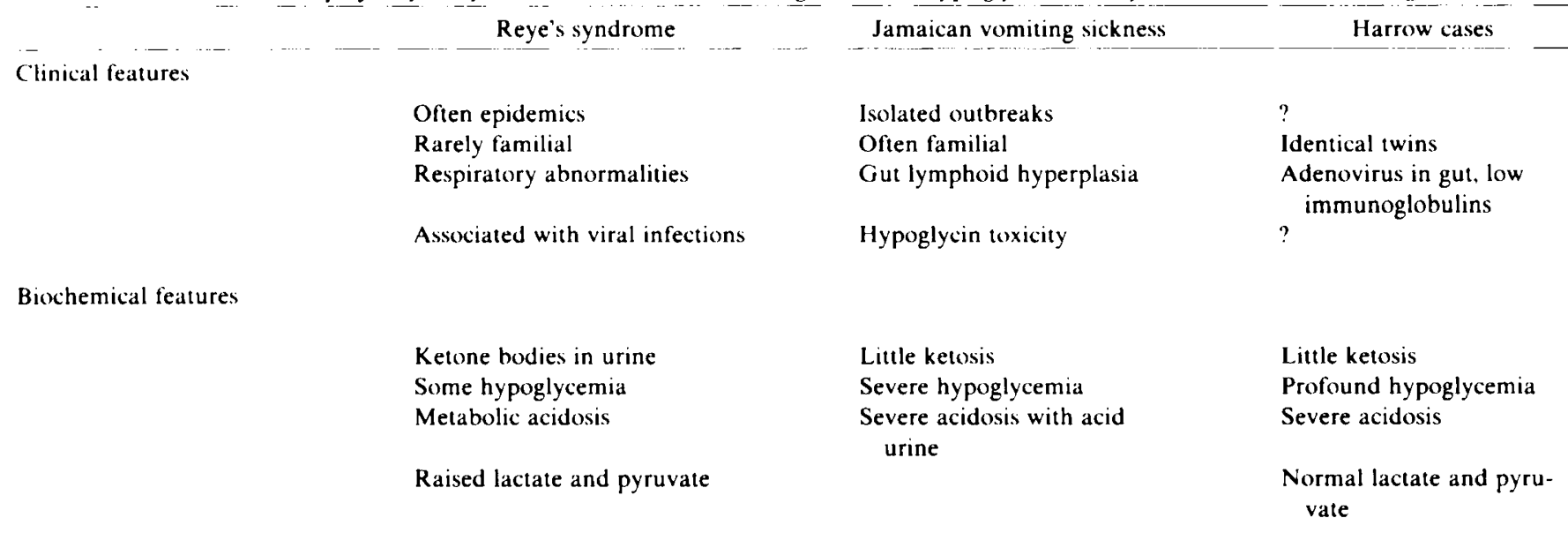

Common features

\footnotetext{
Occur in winter months, vomiting, convulsions, coma death/high mortality, depletion of liver glycogen. small droplet fatly infiltration of liver fand kidney and myocardium in Reye's syndrome and Jamaican vomiting sickness)
}

Methylenecyclopropylacetic acid, pent-4-enoic acid, and hex-4enoic acid, which all contain the $\mathrm{CH}=\mathrm{C}-\mathrm{C}-\mathrm{C}-\mathrm{COOH}$ group that appears to be necessary for their hypoglycemic effects (28), presumably act via their coenzyme $A$ and carnitine esters to inhibit fatty acid $\beta$-oxidation, specific acyl $\mathrm{CoA}$ dehydrogenases, and reactions in which acetyl $\mathrm{COA}$ normally participates to produce the symptoms and biochemical findings observed. The chemical nature of the toxins appears to be critical. and their effects would be expected to vary in detail, as has already been observed for methylenecyclopropylacetic acid and pent-4-enoic acid $(28,29)$. although closely similar in overall effects. Inhibition of fatty acid $\beta$-oxidation and transport leads to depletion of acetyl CoA. decreased availability of NADH, and inhibition of the mitochondrial malate "shuttle" and of acetyl CoA activation of the key enzyme in gluconeogenesis, pyruvate carboxylase (Fig. 4). These effects lead to profound hypoglycaemia, inactivation of the tricarboxylic acid cycle, and depletion of glycogen stores. Fatty acids and lipids accumulate with $\omega$-oxidation of the fatty acids to yield the dicarboxylic aciduria, without ketosis, that is observed. It is possible that some cases of Reye's syndrome are due to potentiation by viral agents of the apparently endogenous inhibitor of green acyl (butyryl) CoA dehydrogenase (13). 
Table 3. Urinary organic acids in Reve's syndrome, Jamaican vomiting sickness, and the Harrow: England cases

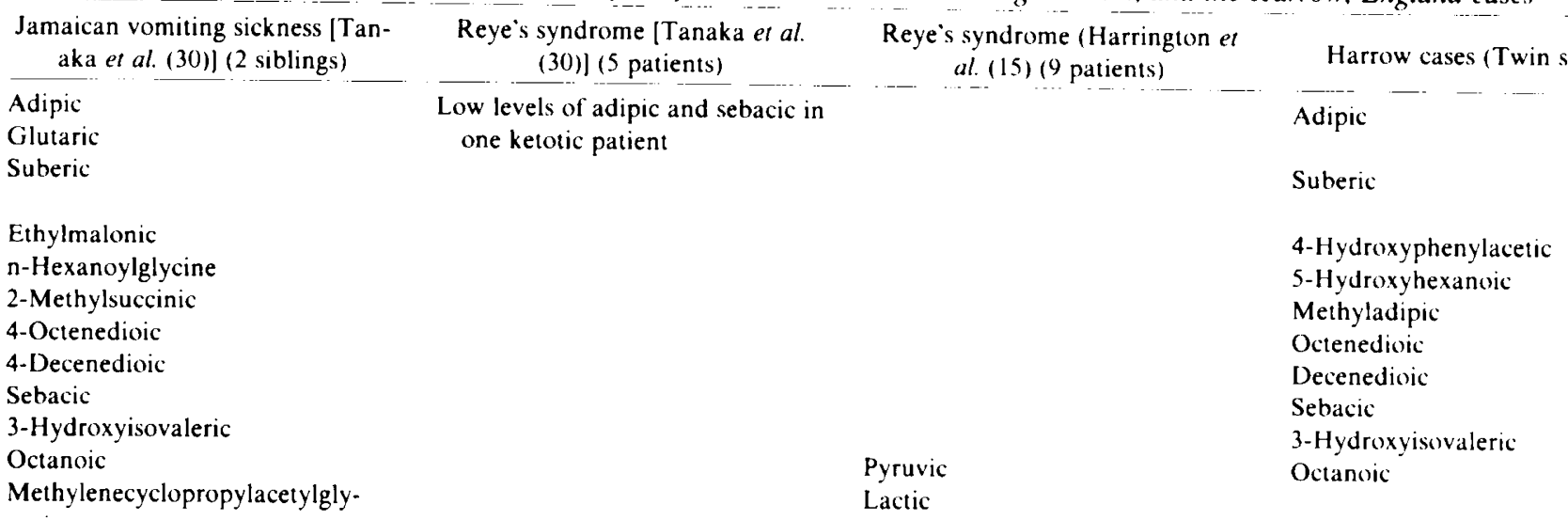

cine

Pyruvic

Normal levels of tricarboxylic acid Low or absent levels tricarboxylic cycle acids.

\author{
acid cycle acids, aldonic acids
}

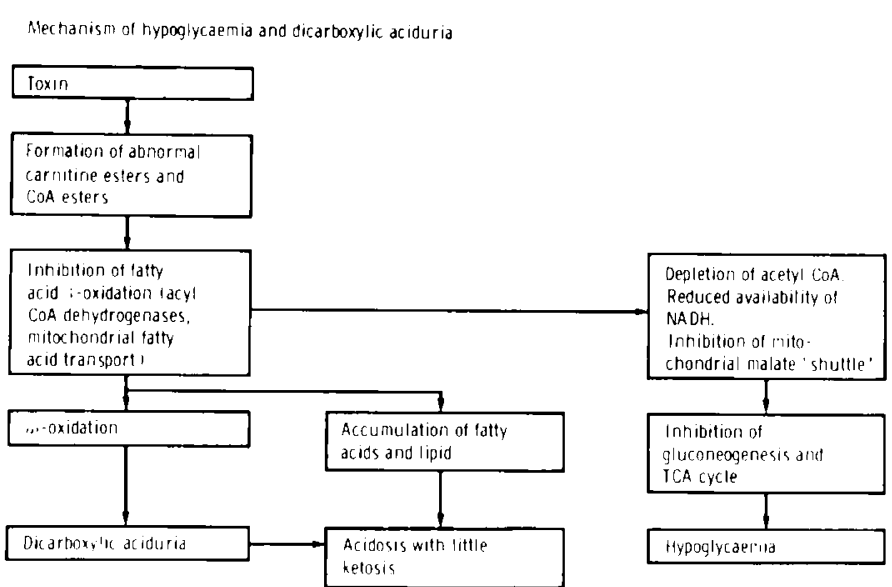

lig. 4. Proposed mechanisms of action of the hypoglycemic analogues of methylenecyclopropylacetic acid.

The observations on the present cases have provided some insight into the biochemistry underlying two cases of apparent Reye's syndrome and have demonstrated the occurrence of a syndrome in England with close clinical and biochemical similarities to Jamaican vomiting sickness. It is apparent that some cases of Reye's syndrome at least are probably due to similar causes, that is organic acid chemical toxins related to hypoglycin and its metabolites and that it is essential to examine urinary organic acids at presentation in such cases to improve further the knowledge of the etiology of Reye's syndrome in infants.

\section{REFERENCES AND NOTHS}

1. Aivathurai. J. E. J.: Reye's syndrome and viral ketwacidosis. Lancet. ?: 1253 (1976).

2. Aprille. J. R.: Reyes syndrome: patient serum alters mitochondial function and morphology in vitro. Science (Wash. D. (.). $19^{?}: 9(1) \times(1977)$.

3. Bieberdorf. F. A.: Differential approach to acute diarrhoeal syndromes. In: J. M Diestschy: Disorders of the Gastrointestinal Tract. Disorders of the Liver. Nutritional Disorders. p. ox (Grune \& Siratton. Inc. New York. 1976)

4. Bjorkman. L.. McLean. C.. and Steen. (i.: Organic acids in urine from human newborn. (lin. (hem.. 2249 (1976)

5. (halmers, R. A.. unpublished data.

6. Chalmers, R. A., and Lawson. A. M.: Identification of S-hvdroxy hexanoic acid in the urine of twin siblings with a Reve's-like syndrome associated with dicarhoxylic aciduria and hypoglycaemia and with similarities to Jamaican vomiting sickness. Biomed. Mass Spectrom., 0: 444 (1974).

7. Chalmers, R. A.. Lawson. A. M.. and Borud. O.: Gas chromatographic and mass spectrometric studies on urinary organic acids in a patient with congenital lactic acidosis due to pyruvate decarboxvlase deticiency. (lin. ( him Acta $\rightarrow 7$ $117(1977)$.
X. Chalmers. R. A.. Lawson. A. M., Whitelaw, A.. and Purkiss, P.: Organic acids in Reye's-like syndrome: similarities with Jamaican vomiting sickness. Lancet. $I$ : $1156(1977)$.

9. (halmers. R. A.. and Watts. R. W. E.: The quantitative extraction and gas-liquid chromatographic determination of organic acids in urine. Analyst. 47 ? 458 (1972).

11). Colon, A. R., Ledesma. F.. Pardo, V., and Sandberg. D. H.: Viral potentiation of chemical toxins in the experimental syndrome of hypoglycaemia. encephalopathy, and visceral fatty degeneration. Digestive Dis.. $19: 1091$ (1974)

11. Corey. I... Ruhin. R. M.. Hattwick. M. A. W.. Noble. (;. R.. and Cassidy, E.: A nationwide outbreak of Reyes syndrome. Its epidemiologic relationship to influenza B. Am. J. Med.. 61: 615 (1976).

12. Editorial. Lancet. ?: $1 \times 3(1476)$.

13. Engel. P. ('.. and Massey. V.: (ireen butyryl-coenzyme A dehydrogenase. An enzyme-acyl-coenzyme A complex. Biochem. J.. 125: 889 (1971).

14. Gregersen. N.. Lauritzen. R.. and Rasmussen. K.: Suberylglycine excretion in the urine from a patient with dicarboxylic aciduria. (lin. ( him. Acta. 7): 417 (1976).

15. Harrington. W.. Liu, A-S.. Lonsdale. D., and lgou. D.: Urinary organic acid profiles of Reye's syndrome. Clin. (him. Acta. 74: 247 (1977).

16. Healy, M J R. (halmers. R. A and Watts, R W . . Reduction ol datis from the automated gas-liquid chromatographic analysis of complex extracts from human biological nluids using a digital electronic integrator and an off-line computer program. J. ( hromatogr.. $\&^{7}: 365$ (1973).

17. Huttenlocher, P. R.. and Trauner. D. A.: Reye's syndrome in infancy. Pediatrics. $0 ?: 84(1978)$.

1X. Karpati. G.. Carpenter, S.. Engel. A. G.. Watters, (i., Allan, J.. Rothman. S. Klassen. (B.. and Mamer, O. A.: The syndrome of systemic carnitine deficiency. Neurology. 25: 16 (1975).

19. Lawson. A. M.. (halmers. R. A., and Watts. R. W. E.: Urinary organic acids in man I. Normal patterns. (lin. ('hem.. 22: 1283 (1976).

20. Lindstedt. S.. Norberg. K.. Steen. (.. and Wahl. E.: Structure of some aliphatic dicarboxylic acids tound in the urine of an infant with congenital lactic acidosis. ('lin. ('hem.. 22: 1330)(1976)

21. Loury. M. F.: Reye's syndrome. Its relationship to Jamaican vomiting sicknes In E. A. Kean: Hypoglycin p. 45 (Academic Press. New York. 1975)

22. Mowat. A. P.: Incephalopathy and tatty degeneration of viscera. Reves sindrome. Arch I) is (hild 45.411 (1973)

23. Mullen. P. W.. Immunopharmacological (onsideration in Reye's sndrome: A powsible xenobiotic initiated disorder? Biochem. Pharmatcol.. ?': 145 (1478).

24. Olson. L. ( . Bourgeots, (. H., Citton. R. B.. Haricul. S., Grossman. R. A.. and Smith. T. J.: Kncephalopathy and fatty degeneration of the viscera in north eastern Thailand. (linical syndrome and epidemologg. Pediatrics, f $^{-} 707$ (1971).

25. Petterson. J. E.. Jellum. E... and LIdjarn. L.: The occurrence of adipic and suberic acid in urine from ketotic patients. (lin. (him. Acta. 3s: 17 (1972).

26. Privrembel. H. Wendel. V.. Becker. K.. Bremer. H. J.. Bruinvis, L... Kelting. D and Wadman. S. K.: (ilutaric aciduria type II: report on previously undercribed metabolic disorder. (lin. ( hom. Acta, of): 227 (1976)

27. Reve. R. D. K.. Morgan. ( $i$.. and Baral, J.: Encephalopaths and fatty degeneration of the viscera. A disease entity in childhood. Lancet. $2.744(196,3)$

2X. Sherratt. H. S. A and Osmundsen. H.: (iommentary on the mechanisms of some pharmacological actions of the hypoglycatemic toxins hypoglycin and pent-4. enoic acid. A way out of the present confusion. Biochem. Pharmacorl. 25: 74. (1976).

29. Tanaka. K.. Issethacher. K. J.. and Shih. V.: Isovaleric and n-methylhusric acidurias induced try hypoglycin $\mathrm{A}$ : mechanisms of Jamaican wometing sickness Science (Wash. D) ( $)$, I : $: 04(1972)$.

30. Tanaka. K. Kean. E. A. and Johnson. B. Jamaican womiting sicknes. Biochem- 
ical investigation of two cases. N. Engl. J. Med.. 245: 461 (1976).

31. Tanaka. K.. Mantagos. S.. (jenel, M.. Seashore. M. R.. Billings, B. A.. and Baretz. B. H.: New defect in fatty acid metabolism with hypoglycaemia and organic aciduria. Lancet. $2: 986$ (1977).

32. Tanaka, K. Ramsdell. H. S.. Baretz, B. H., Keefe, M. B.. Kean. E. A., Johnson. B.: Identification of ethylmalonic acid in urine of two patients with the vomiting sickness of Jamaica. (lin. ( him. Acta, o4: 105 (1976).

33. Thaler M M. Bruhn. R. W.. Applebaum. M. N.. and Goodman. J.: Reye's syndrome in twins. J. Pediatr. $638(1970)$.

34. Tracev, B. M.. (hu, M. D.. Lawson. A. M.. and Chalmers, R. A.: (unpublished data).

35. van Callie, M. Morin. C. L.. Roy. ('. ('., Cieoffroy. (;.. and McLaughlin. B.: Reve's syndrome: relapses and neurological sequelae. Pediatrics, 59: 244 (1977).
36. Whitelaw, A.. Davies, H., and Parry. J.: Electron microscopy of fatal adenovirus gastroenteritis. Lancet. $I: 36$ ( $(1977$ ).

37. The studies on these patients were approved by the Ethical Committee of Northwick Park Hospital and (linical Research Centre.

38. The authors thank Drs. K. Tanaka., S. K. Wadman. and M. Duran, and $N$ Gregersen for stimulating discussions on these cases and possible origins of 5 hydroxyhexanoic acid. F. D. Raymond. M. J. Madigan. and M. D. Chu provided skilled technical assistance.

39. Requests for reprints should be addressed to: Ronald A. Chalmers, PhD. Division of Inherited Metabolic Diseases. M. R. (C. Clinical Research Centre. Watford Road. Harrow. HAI 3UJ, United Kingdom.

40. Received for publication July 11, 1979.

41. Accepted for publication January 3. 1980

Copyright (1) 1980 International Pediatric Research Foundation. Inc

Printed in U.S.A (c) $31-3998 / 80 / 1410-1097 \$(12.00) / 0$ 